\section{Acute Reversal of Clopidogrel-Related Platelet Inhibition Using Methyl Prednisolone in a Patient with Intracranial Hemorrhage}

Clopidogrel and ticlopidine are agents that irreversibly inhibit adenosine diphosphate (ADP)-induced platelet aggregation. ${ }^{1}$ Interaction of ADP with the P2Y1 receptor of the platelet induces platelet shape change, reversible aggregation, initial glycoprotein IIb/IIIa activation, phospholipase $\mathrm{C}$ activation, and calcium flux. ${ }^{1}$ Thienopyridines, including clopidogrel and ticlopidine, are selective noncompetetive inhibitors of the P2Y12 receptor. Regular use of clopidogrel (75 mg daily) can produce $40 \%-50 \%$ inhibition of ADP-induced platelet aggregation. ${ }^{1,2}$ Platelet function recovers gradually 3-5 days after drug withdrawal. ${ }^{2}$ In some circumstances, acute reversal is required, but no specific agent is documented except platelet transfusion. ${ }^{2}$ The increased bleeding time associated with ticlopidine can be normalized within 2 hours by intravenous injection of $20 \mathrm{mg}$ of methyl prednisolone, ${ }^{2}$ but, to our knowledge, no such data are available for clopidogrel, despite the similar mechanism of antiplatelet activity.

We report our experience with a 39-year-old woman with traumatic bilateral internal carotid artery dissections following a traffic crash. Multiple episodes of transient left retinal ischemic events and near-syncope prompted investigations, which demonstrated severe bilateral narrowing of the high cervical internal carotid arteries on CT angiography. The patient received a 300-mg bolus of clopidogrel and was started on daily doses of 75-mg clopidogrel with $325 \mathrm{mg}$ of aspirin, and an interventional procedure was scheduled for the following day. The left internal carotid artery was recontructed with stent placement with no residual narrowing and good distal flow on postprocedural images. Four hours postprocedure, the patient developed partial aphasia, and a CT scan demonstrated a minimal temporal subarachnoid hemorrhage. It was decided that antiplatelet agents should not be reversed by platelet transfusion, to avoid the risk of stent thrombosis. The patient remained neurologically unchanged, but a repeat CT scan in the morning (18 hours after the procedure) demonstrated new hematoma in the left temporal lobe.

A platelet function assay (PFA-100, Dade Behring, Deerfield, Ill) using platelet closure time demonstrated a value above 300 seconds (the maximum measurable value by the instrument) following activation by $10 \mu \mathrm{g}$ of epinephrine. The closure time is a measure of platelet activation in response to high shear stress and agonists like epinephrine. Partial reversal of clopidogrel was attempted by intrave- nous administration of $25 \mathrm{mg}$ of methyl prednisolone. Platelet closure time after 4 hours was 175 seconds by using activation with epinephrine, indicating greater platelet adhesion and aggregation. Both aspirin and clopidogrel were discontinued. Serial CT scans performed on days 2 and 3 demonstrated no change in the size of the intraparenchymal hemorrhage. On postprocedure day 4, the patient was restarted on aspirin. Platelet closure time on day 4 was 191 seconds by using epinephrine activation. At 1-month evaluation, the patient had no neurologic deficits or recurrence of retinal ischemic or near-syncopal events.

We observed rapid reduction in the platelet inhibition following intravenous injection of methyl prednisolone in a patient receiving clopidogrel and aspirin. Although the beneficial effect of methyl prednisolone may not be specific to ADP-mediated platelet inhibition, we think that previously reported data regarding other thienopyridines are consistent with this assumption. Acute reversal of clopidogrelmediated platelet inhibition by using methyl prednisolone is important because of the increasing use of clopidogrel and the concomitantly increasing rate of strokes in patients using clopidogrel. ${ }^{3}$ Rapid reversal of antiplatelet activity may prevent hematoma expansion ${ }^{4}$ and consequent mortality in such patients. ${ }^{5}$

\section{References}

1. Hassan AE, Zacharatos H, Suri MF, et al. Drug evaluation of clopidogrel in patients with ischemic stroke. Expert Opin Pharmacother 2007;8:2825-38

2. Qureshi AI, Luft AR, Sharma M, et al. Prevention and treatment of thromboembolic and ischemic complications associated with endovascular procedures. Part I. Pathophysiological and pharmacological features. Neurosurgery 2000;46:1344-59

3. Qureshi AI, Kirmani JF, Safdar A, et al. High prevalence of previous antiplatelet drug use in patients with new or recurrent ischemic stroke: Buffalo metropolitan area and Erie County stroke study. Pharmacotherapy 2006;26:493-98

4. Tuhrim S. Aspirin-use before ICH: a potentially treatable iatrogenic coagulopathy? Stroke 2006;37:4-5. Epub 2005 Dec 1

5. Saloheimo $\mathrm{P}$, Ahonen M, Juvela S, et al. Regular aspirin-use preceding the onset of primary intracerebral hemorrhage is an independent predictor for death. Stroke 2006;37:129-33. Epub 2005 Dec 1

A.I. Qureshi

M.F.K. Suri

Zeenat Qureshi Stroke Research Center

Department of Neurology University of Minnesota Minneapolis, Minn

DOI 10.3174/ajnr.A1297 